\title{
Shaped pupil coronagraphs for high-contrast imaging with SPICA
}

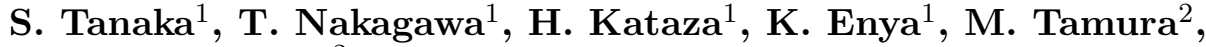 \\ L. Abe ${ }^{2}$, and the SPICA coronagraph team
}

${ }^{1}$ Institute of Space and Astronautical Science, Japan Aerospace Exploration Agency, 3-1-1

Yoshinodai, Sagamihara, Kanagawa, 229-8510, Japan

email:stanka@ir.isas.jaxa.jp

${ }^{2}$ National Astronomical Observatory of Japan, 2-21-1 Osawa, Mitaka, Tokyo, 181-8588, Japan

\begin{abstract}
We present configurations of shaped pupil coronagraphs optimized for a realistic telescope to directly detect extrasolar giant planets in the mid-IR wavelengths. This study is linked to the development of the coronagraphic instrument aboard SPICA (SPace Infrared telescope for Cosmology and Astrophysics). We made a systematic assessment of the performance of the "checkerboard" and "concentric ring" masks, introducing a large central obstruction (C.O.) due to a secondary mirror and its related support spiders. With a small secondary mirror, we also propose a modification to the original symmetrical checkerboard apodization, which enables us to achieve a $10^{-7}$ contrast level at $4.0 \lambda / D$. The transmission through the optimal binary masks exhibits abrupt increases and plateaus as the inner working angle (IWA) is increased. We attribute these properties of binary apodization function to the existence of threshold IWAs that allow large openings in the pupil.
\end{abstract}

Keywords. instrumentation: high angular resolution.

\section{Introduction}

It is planned to launch SPICA, a Japanese cooled infrared telescope with a $3.5 \mathrm{~m}$ diameter primary mirror, in the early 2010s (Nakagawa 2004). One target of the SPICA mission is the direct imaging and spectroscopy of extrasolar giant planets in the mid-IR wavelengths (Abe et al. 2005; Enya et al. 2005; Tamura et al. 2005). The requirement on the SPICA coronagraphic instrument is to realize a contrast of $10^{-6}$ from an IWA of $3-5 \lambda / D$ to an OWA (outer working angle) of $\sim 10 \lambda / D$ during actual operation.

Shaped pupil techniques have important advantages such as the full achromaticity, the simplicity of optical system, and the ease of fabrication. However, few previous studies on binary pupils took C.O.s in the telescope pupil into account: most of them were for obstruction-free telescopes like an off-axis one. Hence, they cannot be applied directly to more realistic general-purpose telescopes, including SPICA, whose pupil functions are complicated due to the existence of a large secondary mirror and its related support spiders. The main objective of this paper is to find optimal binary apodization functions that maintain tight IWAs for a telescope pupil with a large C.O. We here require ideal performance of $10^{-7}$ contrast level. We also reveal the general properties of binary apodization function, from a careful analysis of the behavior of transmission through binary masks according to the values of IWA. 

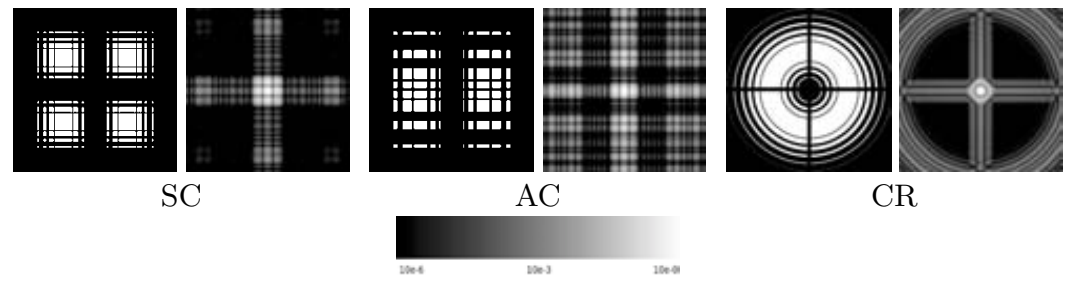

Figure 1. The optimal masks for $\alpha_{\mathrm{s}}=0.14$ and their associated PSFs. The side lengths of the mask images correspond to the pupil diameter. The PSF images show the central $40 \lambda / D \times 40 \lambda / D$ region in logarithmic scale. $\alpha_{\text {spi }}$ is 0.0228 for the AC and CR masks.

\section{Masks for a pupil with a large C.O.}

\subsection{Configuration}

A possible solution to overcome the obstacle presented by C.O.s is the use of either the concentric ring masks or the checkerboard masks, both proposed recently by Vanderbei et al. $(2003,2004)$. We considered three types of configurations based on those masks. The first one is the "symmetrical checkerboard" (SC) masks whose two aperture functions along $x$ and $y$ directions, $A_{x}(x)$ and $A_{y}(y)$, have the same large C.O. corresponding to the secondary mirror (with diameter $\alpha_{\mathrm{s}} D, D$ is the primary diameter). Secondly, we modified the constraint condition on $A_{y}(y)$ into a looser one: a C.O. corresponding to a spider of width $\alpha_{\mathrm{spi}} D$, not to $\alpha_{\mathrm{s}} D$. The benefit derived from this modification is that it allows us to shift the contrast requirement on $A_{x}(x)$ to $A_{y}(y)$, resulting in an improvement in the IWA. We call them the "asymmetrical checkerboard" (AC) masks. The last one is the concentric ring (CR) masks with cross-shaped spiders. The C.O. by the secondary mirror was taken into account in the optimization processes while the spiders were unavoidable.

We simulated large secondary mirrors with various diameters: $\alpha_{\mathrm{s}}=0.1,0.14,0.2$, and 0.24 . The width of the spider arms is fixed at $\alpha_{\mathrm{spi}}=0.0228$. We required that the mask transmission, defined as the ratio of the total open area to a full circular pupil, be higher than $15 \%$ and discarded the solutions that did not meet this criterion. Solutions whose IWAs are larger than $6.0 \lambda / D$ were excluded. Figure 1 shows examples of the optimal mask shapes we found and their PSFs.

\subsection{Comparison of the performance}

Table 1 shows that the AC masks have the tightest IWAs in almost all the cases. For $\alpha_{\mathrm{s}}<0.15$, we obtain an IWA of $4.0 \lambda / D$ with acceptable transmission. However, since the IWA of the checkerboard masks monotonically increases as the C.O. gets larger, the CR masks, whose performance is rather insensitive to the secondary mirror size, become a contender for $\alpha_{\mathrm{s}} \geqslant 0.2$. The greater transmission of the CR masks, which is more than twice that of the checkerboard masks, could be the dominant factor that decides the supremacy in this regime. Figure 2 compares the total dark area obtained by the three configurations. In the cases of $\alpha_{\mathrm{s}} \leqslant 0.14$, the AC masks have a significantly wider dark area inside the radius of $\sim 6.5 \lambda / D$. However, the superiority of the $\mathrm{AC}$ masks to the $\mathrm{CR}$ masks is less obvious when $\alpha \geqslant 0.2$. In conclusion, the AC masks are promising due to the larger dark area for $\alpha_{\mathrm{s}} \lesssim 0.15$. For larger C.O.s $\left(\alpha_{\mathrm{s}} \gtrsim 0.15\right)$, on the other hand, the CR masks provide the best compromise between search space and mask transmission.

Since the spider effects cannot be avoided with the CR masks, we are also interested in whether their performance is sensitive to the spider widths. When the spider arms are wider, the contrast gap between the cross-shaped diffraction halos and the PSF center decreases. Although the total dark area does not change much according to the arm 
Table 1. Optimal solutions found for the symmetrical checkerboard (SC), asymmetrical checkerboard $(\mathrm{AC})$, and concentric ring $(\mathrm{CR})$ masks. The spider arm widths and the total contrast are fixed at $\alpha_{\mathrm{spi}}=0.0228$ and $10^{-7}$, respectively. The IWAs and OWAs are in $\lambda / D$ unit.

\begin{tabular}{l|llll|llll|llll}
\hline$\alpha_{\mathrm{s}}$ & Mask & IWA & OWA & Trans. & Mask & IWA & OWA & Trans. & Mask & IWA & OWA & Trans. \\
\hline 0.1 & SC_1 & $4 \sqrt{2}$ & $15 \sqrt{2}$ & $23 \%$ & AC_1 & 4.0 & 12.3 & $20 \%$ & CR_1 & 4.7 & 17.0 & $48 \%$ \\
0.14 & SC_2 & $4 \sqrt{2}$ & $13.5 \sqrt{2}$ & $23 \%$ & AC_2 & 4.0 & 11.4 & $18 \%$ & CR_2 & 4.7 & 15.0 & $48 \%$ \\
0.2 & SC_3 & No Solution Found & AC_3 & 5.0 & 11.4 & $15 \%$ & CR_3 & 4.7 & 12.0 & $38 \%$ \\
0.24 & SC_4 & \multicolumn{2}{c}{ No Solution Found } & AC_4 & 5.4 & 11.4 & $17 \%$ & CR_4 & 6.0 & 12.0 & $39 \%$ \\
\hline
\end{tabular}
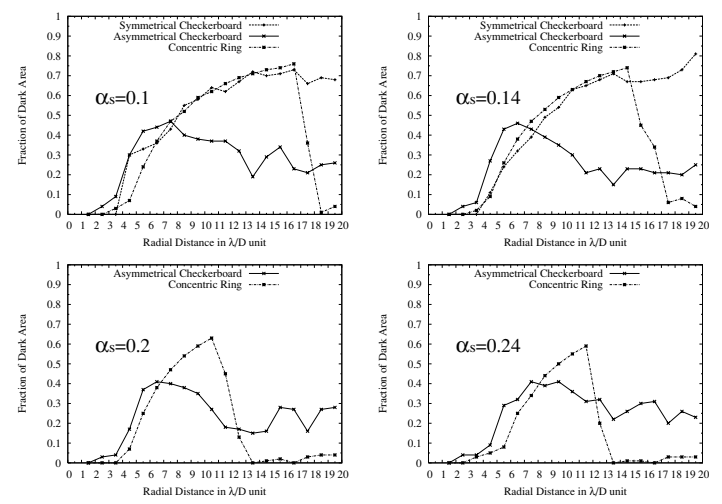

Figure 2. - The fraction of dark area (dimmer than the PSF core by 6 orders of magnitude or more) within a $1.0 \lambda / D$-wide annuli each of which has the radius of $1.5,2.5, \ldots$, and $19.5 \lambda / D$.
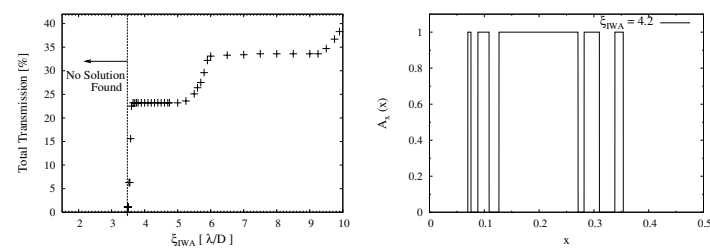

Figure 3. - left: Dependence of the mask transmission on the IWA (see text for the definition of the parameters). Under the current configuration, the maximum transmission available is 41 \% right: The cross-sectional cut of the optimal aperture functions, $A_{x}(x)$, for $\xi_{\text {IWA }}=4.2 \lambda / D$.

width, therefore, it is still preferable to use narrower spider arms in view of total amount of light that gets into the main lobe of the PSF.

\section{Behavior of Binary Aperture Functions}

In this section, we discuss the relationship between the IWA and mask transmission. Although the following analysis can be extended to the AC and CR masks, we limit our discussion to SC masks with a 2-D OWA of $10 \sqrt{2} \lambda / D$ (the corresponding 1-D OWA is $\left.\xi_{\text {OWA }}=10 \lambda / D\right)$ and total contrast of $10^{-7}$. We also impose a $14 \%$ C.O. Figure 3 (left) shows that the transmission curve exhibits a stepwise behavior. There are two abrupt increases at 1-D IWA of $\xi_{\text {IWA }}=3.6 \lambda / D$ and around $\xi_{\text {IWA }}=6.0 \lambda / D$, and also two plateaus at transmission of $23 \%$ and $34 \%$.

Fig. 3 also shows the cross-sectional cut of the aperture functions, $A_{x}(x)$, at $\xi_{\text {IWA }}=$ $4.2 \lambda / D$. From the Fourier transform of such multiple boxcar functions, we obtain the 
brightness contrast ratio realized at point $\xi$ as follows:

$$
C(\xi)=\left\{\frac{\sum_{j} T_{j}(\xi) \cdot S_{j}(\xi)}{\sum_{j} W_{j}}\right\}^{2} .
$$

$T_{j}(\xi)$ refers to the trigonometric function term $\cos \left(2 \pi \xi X_{j}\right)$ depending on $X_{j} \equiv\left(x_{2 j}+\right.$ $\left.x_{2 j-1}\right) / 2$, the position of the midpoint of the $j$-th opening, and $S_{j}(\xi)$ denotes the sinc function term $W_{j} \sin \left(\pi \xi W_{j}\right) /\left(\pi \xi W_{j}\right)$ depending on $W_{j} \equiv x_{2 j}-x_{2 j-1}$, its width.

The denominator in the equation above, $\sum_{j} W_{j}$, represents the transmission of this apodization. While wider openings provide better transmission, the openings have to satisfy the requirements imposed by the values of the IWA, OWA, and contrast. We point out here the factors that constrain the widths of openings in the pupil. The first one is the value $\left|\sum_{j} T_{j}(\xi) S_{j}(\xi)\right|$, which must be very small in the dark region. In order to ensure this, all $T_{j} S_{j}$ should have roughly similar amplitudes inside the dark region under consideration. Any component which has significantly larger $\left|T_{j} S_{j}\right|$ in the dark region than those of the others is thus prohibited. The second factor is the extent of the dark region, or the distance from the IWA to the OWA. A large number of openings (i.e., a denser set of $X_{j}$ ) are needed to enlarge the dark region. The inner and outer openings are especially critical for the depth of the null. If the amplitude contributed by inner openings (with small $X_{j}$ ) is large (with large $W_{j}$ ), we need many other components with short $T_{j}$ periods (large $X_{j}$ ) to compensate for it in the dark region. Therefore, it is necessary that the amplitudes contributed by the inner openings are small, and this is realized by narrower openings $\left(\right.$ small $W_{j}$ ). Components with short periods (large $X_{j}$ ) are also important because higher frequency components are used to finely tune the summation $\Sigma_{j} T_{j} S_{j}$, and thereby produce a deeper null. A wide opening (with large $W_{j}$ ) at the outermost region of the pupil causes a large inward shift of $X_{j}$, resulting in a loss of higher frequencies. Thus, in general, the openings near the inner and outer edges of the pupil become narrower than those in the intermediate region (see Fig. 3).

The saturation in the transmission at IWAs of $4 \sim 5 \lambda / D$ is an important property which suggests that we ought to choose a solution just after the first transmission jump.

\section{Summary}

We discussed the dependence of coronagraphic performance of some binary masks, taking into account the effects from the secondary mirror and cross-shaped spiders. When the secondary mirror is rather small, we obtain a contrast level of $10^{-7}$ even at an IWA of $4.0 \lambda / D$. The transmission curve of the optimal binary apodization function exhibits a rapid increase near a certain threshold value of the IWA and becomes saturated after that, indicating that for practical use we should choose the function at the onset of saturation.

\section{References}

Abe, L., et al. 2005, this conference

Enya, K., et al. 2005, this conference

Nakagawa, T. 2004, Advances in Space Research, 34, 645

Tamura, M., Abe, L., HiCIAO team, SPICA coronagraph team, \& JTPF working group. 2005, this conference

Vanderbei, R. J., Spergel, D. N., \& Kasdin, N. J. 2003, ApJ, 590, 593

Vanderbei, R. J., Kasdin, N. J., \& Spergel, D. N. 2004, ApJ, 615, 555 